\title{
The possibilities of compressed-sensing-based Kirchhoff prestack migration
}

\author{
Ali Aldawood ${ }^{1}$, Ibrahim Hoteit ${ }^{1}$, and Tariq Alkhalifah ${ }^{1}$
}

\begin{abstract}
An approximate subsurface reflectivity distribution of the earth is usually obtained through the migration process. However, conventional migration algorithms, including those based on the least-squares approach, yield structure descriptions that are slightly smeared and of low resolution caused by the common migration artifacts due to limited aperture, coarse sampling, band-limited source, and low subsurface illumination. To alleviate this problem, we use the fact that minimizing the L1-norm of a signal promotes its sparsity. Thus, we formulated the Kirchhoff migration problem as a compressed-sensing (CS) basis pursuit denoise problem to solve for highly focused migrated images compared with those obtained by standard and least-squares migration algorithms. The results of various subsurface reflectivity models revealed that solutions computed using the CS based migration provide a more accurate subsurface reflectivity location and amplitude. We applied the CS algorithm to image synthetic data from a fault model using dense and sparse acquisition geometries. Our results suggest that the proposed approach may still provide highly resolved images with a relatively small number of measurements. We also evaluated the robustness of the basis pursuit denoise algorithm in the presence of Gaussian random observational noise and in the case of imaging the recorded data with inaccurate migration velocities.
\end{abstract}

\section{INTRODUCTION}

Seismic imaging using Kirchhoff-type integrals, which is based on high-frequency asymptotic solutions to the wave-equation, is a powerful tool to map the data recorded on the surface of the earth into representative subsurface structures. However, a major draw- back of standard Kirchhoff migration is that it suffers from migration artifacts, which results in a blurred depiction of the true subsurface reflectivity distributions. This effect is primarily caused by the limited acquisition aperture, coarse source-receiver sampling, band-limited source wavelet, and low subsurface illumination (Nemeth et al., 1999; Tang, 2009).

Least-squares migration (LSM) has the potential to retrieve the subsurface reflectivity series more accurately than standard migration. LSM is used to reduce the migration artifacts and enhance the spatial resolution of the migrated image (LeBras and Clayton, 1988; Nemeth et al., 1999). It provides an approximate solution for the subsurface reflectivity as demonstrated by the linearized waveform inversion (Snieder et al., 1988; Landa et al., 1989; Crase et al., 1990; Roth and Tarantola, 1992).

Adding a regularization term to the objective function may suppress the migration artifacts and sharpen the subsurface reflectivity distribution (Fomel and Guitton, 2006). Most regularization techniques assume that the inverted model parameters are smooth and continuous (Aster et al., 2005). However, because the reflectivity distribution (i.e., our model parameter) is sparse and discontinuous, we could formulate the Kirchhoff migration problem as a compressed-sensing (CS) problem, which could be solved using L1minimization algorithms.

CS, or sparse recovery, is an emerging field that is widely used to solve linear systems when the desired signal is sparse (Donoho, 2006; Eldar and Kutyniok, 2012). It relies on the fact that many signals or images can be well-approximated by a linear combination of suitable basis or dictionaries (e.g., Fourier, or wavelets) with only a small number of nonzero coefficients (Donoho, 2006). JPEG and MP3 are typical examples of these lossy compressions in which only large basis coefficients are stored and the rest are set to zero (Pennebaker and Mitchell, 1992; Taubman and Marcellin, 2002).

The power of CS lies in its ability to reconstruct a compressed version of the original signal while requiring only a small number of measurements. Clearly, this could lead to a very important saving in data collection and computational time. For example, the

\footnotetext{
Manuscript received by the Editor 18 July 2013; revised manuscript received 16 February 2014; published online 8 May 2014.

${ }^{1}$ King Abdullah University of Science and Technology, Physical Sciences and Engineering Division, Thuwal, Saudi Arabia. E-mail: ali.aldawood@kaust.edu .sa; ibrahim.hoteit@kaust.edu.sa; tariq.alkhalifah@kaust.edu.sa.

(C) 2014 Society of Exploration Geophysicists. All rights reserved.
} 
implementation of the CS technology resulted in a speedup factor of seven times in obtaining a pediatric magnetic resonance image while preserving the diagnostic quality (Lustig et al., 2005, 2006; Trzasko and Manduca, 2009).

In this paper, we first present the theory of LSM. We next formulate the migration problem as a basis pursuit denoise (BPDN) problem (i.e., L1-minimization problem) and solve it to try to retrieve the sparsest reflectivity distribution that explains the recorded data. The objective of our work is to spatially focus the migrated image and reduce the smearing effect caused by least-squares minimization. We also study whether we can accurately recover a highly resolved subsurface image using a smaller number of measurements while deploying fewer sources and receivers. We finally study the robustness of the CS approach focusing on the retrieval of spatially focused migrated images with reduced migration artifacts in the presence of white Gaussian random noise and in the case of migrating the data using erroneous migration velocity.

\section{LEAST-SQUARES MIGRATION}

The Kirchhoff forward-modeling operator $\mathbf{L}$ maps the subsurface reflectivity distribution to scattered seismic data. Generating synthetic seismic data by Kirchhoff modeling can be represented by a matrix-vector multiplication as

$$
\mathbf{d}=\mathbf{L m},
$$

where $\mathbf{d}$ is the modeled scattered seismic data and $\mathbf{m}$ is the true subsurface reflectivity distribution. A standard migrated image is obtained by applying the adjoint of the forward-modeling operator (Claerbout, 1992):

$$
\mathbf{m}_{\text {mig }}=\mathbf{L}^{\mathbf{T}} \mathbf{d}
$$

where $\mathbf{m}_{\mathbf{m i g}}$ is the migrated image. Substituting the expression of $\mathbf{d}$ in equation 1 into equation 2 yields

$$
\mathbf{m}_{\text {mig }}=\mathbf{L}^{\mathbf{T}} \mathbf{L m}
$$

where $\mathbf{L}^{\mathbf{T}} \mathbf{L}$ is known as the model resolution matrix and reflects the amount of smearing in the seismic image resulting from many factors, including limited recording aperture. The physical interpretation of the model resolution matrix is that the standard migrated image is a linear combination of the true reflectivity model. The Kirchhoff forward operator and its corresponding adjoint operator in their explicit discrete form are given as

$$
\begin{aligned}
& \mathbf{d}(s, g, t)=[\mathbf{L} \mathbf{m}]_{s, g, t}=\beta \sum_{\mathbf{x}} w\left(t-\tau_{s \mathbf{x}}-\tau_{\mathbf{x} g}\right) \mathbf{m}(\mathbf{x}), \\
& \mathbf{m}(\mathbf{x})=\left[\mathbf{L}^{\mathbf{T}} \mathbf{d}\right]_{\mathbf{x}}=\sum_{s} \sum_{g} \sum_{t} w\left(t-\tau_{s \mathbf{x}}-\tau_{\mathbf{x} g}\right) \mathbf{d}(s, g, t),
\end{aligned}
$$

where $s, g, \mathbf{x}$, and $t$ are the source position, receiver position, trial image point, and time sample, respectively. $\tau_{s \mathbf{x}}$ is the traveltime from the source position $s$ to the trial image point $\mathbf{x}$. Similarly, $\tau_{\mathbf{x} g}$ is the traveltime from the trial image point $\mathbf{x}$ to the receiver position $g$. The symbol $\beta$ is an amplitude factor that describes the geo- metrical spreading of the wave. As one may note, the summation is over the model space $\mathbf{x}$ for modeling seismic data whereas it is over the data space $s, g$, and $t$ for migrating recorded data. The migration operators are adjoint operators of the forward-modeling ones because they undo the time and phase shifts of the modeling operators but do not divide the color (i.e., account for the amplitude) (Claerbout and Fomel, 2008).

LSM yields an approximate reflectivity image that minimizes the sum of the squares of the residual vector. Mathematically, the unconstrained objective function is defined as

$$
J=\|\mathbf{L m}-\mathbf{d}\|_{2}=\mathbf{m}^{\mathbf{T}} \mathbf{L}^{\mathbf{T}} \mathbf{L} \mathbf{m}-2 \mathbf{m}^{\mathbf{T}} \mathbf{L}^{\mathbf{T}} \mathbf{d}+\mathbf{d}^{\mathbf{T}} \mathbf{d} .
$$

The minimizer of this objective function is found by taking the gradient with respect to $\mathbf{m}$ and setting it to zero:

$$
\nabla_{\mathbf{m}} J=\mathbf{L}^{\mathbf{T}} \mathbf{L} \mathbf{m}-\mathbf{L}^{\mathbf{T}} \mathbf{d}=0 .
$$

Hence, a more accurate solution to the migration problem is obtained by solving equation 7 , which is the so-called normal equation:

$$
\mathbf{L}^{\mathbf{T}} \mathbf{L m}_{\text {mig }}=\mathbf{L}^{\mathbf{T}} \mathbf{d}
$$

Gradient-based algorithms are generally used to solve this equation iteratively where data residuals are imaged to obtain model updates. By rearranging the terms in equation 7 , the gradient at every iteration is given by

$$
\nabla_{\mathbf{m}} J=\mathbf{L}^{\mathbf{T}}(\mathbf{L m}-\mathbf{d})=\mathbf{L}^{\mathbf{T}} \mathbf{r},
$$

where $\mathbf{r}$ is the data residual vector. The pseudocode for the gradientdescent algorithm is as follows:

$$
\begin{aligned}
& \text { while }\left(\left\|\mathbf{g}^{\mathbf{k}}\right\| \text { is large }\right) \\
& \mathbf{g}^{\mathbf{k}+\mathbf{1}}=\mathbf{L}^{\mathbf{T}}\left(\mathbf{L m}^{\mathbf{k}}-\mathbf{d}\right) \\
& \mathbf{m}^{\mathbf{k}+\mathbf{1}}=\mathbf{m}^{\mathbf{k}}-\alpha \mathbf{g}^{\mathbf{k}+\mathbf{1}} \\
& k=k+1 \\
& \text { end. }
\end{aligned}
$$

The gradient $\mathbf{g}^{\mathbf{k}}$ is obtained by back-projecting the data residual vector using the adjoint of the Kirchhoff forward-modeling operator. At each iteration $k$, the reflectivity vector $\mathbf{m}$ is updated by moving along the negative gradient direction (i.e., the steepest descent direction) and the scalar quantity $\alpha$ is the step length that determines how much we move along that direction. The step length is calculated using the following formula (Claerbout and Fomel, 2008):

$$
\alpha=\frac{\mathbf{r}^{\mathbf{T}} \Delta \mathbf{r}}{\Delta \mathbf{r}^{\mathrm{T}} \Delta \mathbf{r}}
$$

where $\mathbf{r}=\mathbf{L} \mathbf{m}-\mathbf{d}$ and $\Delta \mathbf{r}=\mathbf{L} \mathbf{\Delta} \mathbf{m}$.

Although LSM suppresses the migration artifacts and helps focus the migrated image, this L2-norm minimization tends to produce a smeared version of the true reflectivity distribution (Avron et al., 2010; Yu et al., 2011). Thus, a major limitation of LSM is that 
it tends to produce images with limited bandwidth partially due to the band-limitedness of the source wavelet (Ribodetti et al., 2011).

\section{COMPRESSED SENSING}

Because the subsurface reflectivity distribution is known to be sparse and discontinuous, CS can be used to produce highly resolved migrated images from the given data where the migration artifacts are significantly compressed. The sparsest reflectivity distribution is found by solving the following L0-optimization problem (Donoho, 2006):

$$
\min \|\mathbf{m}\|_{0} \text { subject to } \mathbf{L m}=\mathbf{d},
$$

where $\|\mathbf{m}\|_{0}$ counts the number of nonzero elements in the vector $\mathbf{m}$. This optimization problem has a nonconvex objective function, and is NP-hard and intractable because it requires an exhaustive combinatorial search to find the solution. Nevertheless, the L0-norm can be relaxed to the L1-norm leading to a convex optimization problem that is computationally tractable (Chen et al., 2001):

$$
\min \|\mathbf{m}\|_{1} \text { subject to } \mathbf{L m}=\mathbf{d} \text {. }
$$

This L1-minimization problem is known as the basis pursuit approach, which is used to reconstruct sparse signals. Chen et al. (2001) show that this L1-optimization problem can be cast as a linear program. However, in the presence of noise, this constraint cannot be exactly satisfied. In other words, there is no feasible $\mathbf{m}$ that perfectly maps to the recorded scattered data $\mathbf{d}$. Hence, we reformulate the basis pursuit problem as a BPDN problem, which is defined as (van den Berg and Friedlander, 2008)

$$
\min \|\mathbf{m}\|_{1} \text { subject to }\|\mathbf{L m}-\mathbf{d}\|_{2}<\sigma,
$$

where the tolerance $\sigma$ is a nonnegative small scalar. Van den Berg and Friedlander (2007) present an algorithm that solves this problem for any tolerance value. They showed that there is a tradeoff curve between the two objectives: minimizing the least-squares data fitting and minimizing the L1-norm of the solution that promotes sparsity. Therefore, giving more weight to the data fitting is only feasible at the expense of getting a less sparse solution and vice versa. This curve is known as the Pareto optimal curve and van den Berg and Friedlander (2008) show that this curve is convex and continuously differentiable over all points of interest. They claim that their rootfinding algorithm can find an arbitrary point on the Pareto optimal curve.

Van den Berg and Friedlander (2007) basically cast the BPDN problem as a problem of finding the root of a single-variable nonlinear equation $\phi(\tau)=\sigma$ parameterizing the Pareto curve. At each iteration, an estimate of $\tau$ is used to define a convex optimization problem (i.e., a LASSO problem) whose solution yields function and of the scatterer. derivative information that are next used by a Newton-based rootfinding algorithm to probe the Pareto curve. The goal is to find the optimal trade-off between the two competing objectives. Van den Berg and Friedlander (2007) propose a spectral projected gradient algorithm (SPGL1) that iteratively solves a sequence of LASSO problems of the form

$$
\min \|\mathbf{L m}-\mathbf{d}\|_{2} \text { subject to }\|\mathbf{m}\|_{1}<\tau .
$$

SPGL1 is a free MATLAB package that implements the van den Berg and Friedlander (2007) algorithm, and we used it to solve the Kirchhoff imaging problem. At each iteration, the algorithm uses the forward-modeling operator in equation 4 to model the scattered data. Then, the adjoint operator in equation 5 is used to back-project the residual vector to compute the gradient direction as in equation 9 and, consequently a gradient-based model update. The new iterate is then projected orthogonally onto the L1-norm unit ball of size $\tau$ to promote the sparsity of the solution.

\section{SINGLE SCATTERER EXAMPLE}

In this example, the grid spacings of this $75 \times 75$ grid are $\Delta x=\Delta z=7 \mathrm{~m}$, and the time sampling is $2 \mathrm{~ms}$. A point scatterer of unit amplitude is placed at the center of the model shown in Figure $1 \mathrm{a}$, and the background velocity model is constant and equal to $1000 \mathrm{~m} / \mathrm{s}$. We generated 75 noise-free shots located at the surface by a Kirchhoff forward-modeling operator using a Ricker wavelet with a peak frequency of $20 \mathrm{~Hz}$. The acquisition geometry and parameters for this example and the other examples are quite a)

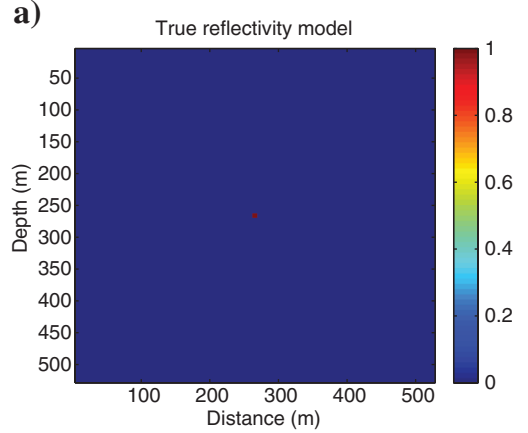

b) Standard migration image

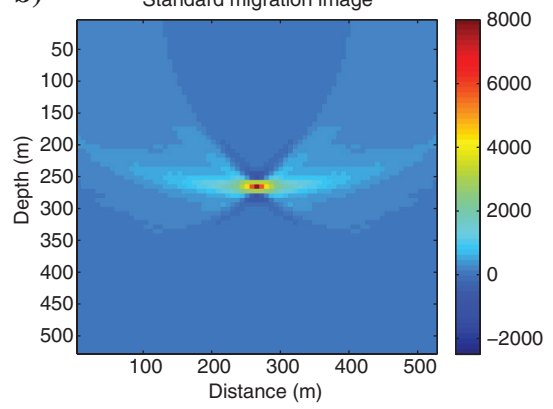

c)

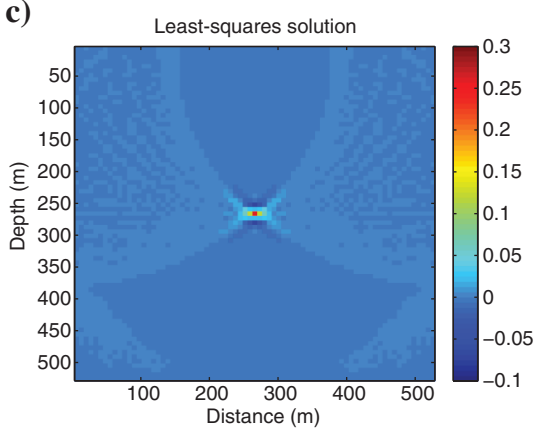

d)

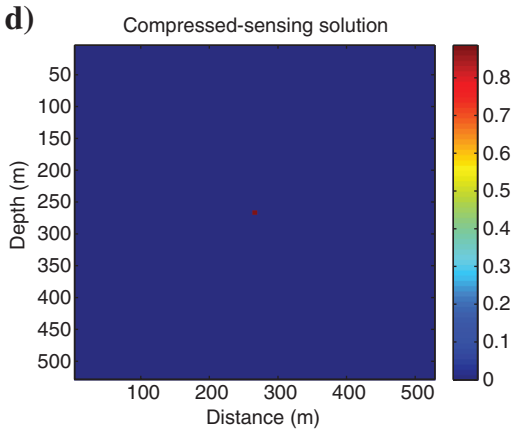

Figure 1. Single scatterer example: (a) true reflectivity model and (b) heavily blurred standard migrated image. (c) The LSM image suppresses the migration smiles, but it results in a smooth depiction of the true reflectivity model. (d) The CS solution dramatically suppresses the migration smiles and give more accurate amplitude information 
similar. The first shot is at the top-left corner, and the last shot is at the top-right corner. The remaining equally spaced shots are placed at the grid points along the earth surface. Similarly, there are 75 equally spaced receivers placed at the grid points along the earth surface.

The standard migrated image is obtained by applying the adjoint of the forward-modeling operator to the shot gathers, and it is shown in Figure 1b. Clearly, the standard migrated image is a blurred version of the true subsurface reflectivity model. Even though the standard migrated image roughly indicates the position of the scatterer, the amplitude of the scatterer is very far from the true one. The migration artifacts (i.e., smiles) deteriorate the quality of the image.

On the other hand, the LSM result in Figure 1c shows a considerable enhancement of the image quality and mitigation of the migration artifacts after 30 gradient descent iterations. Also, this linearized inversion yielded a scatterer amplitude of about 0.25 compared with the true amplitude of 1 . The least-squares solution is still a smooth version of the true subsurface reflectivity model, and that explains the smearing effect in Figure 1c.

Therefore, we try to retrieve a sparse representation of the reflectivity image using CS. The CS solution, obtained by solving the BPDN problem, promotes the sparsity of the subsurface reflectivity distribution as shown in Figure 1d. With a value of $\sigma=1 \times 10^{-4}$, the CS solution shows dramatic removal of the migration artifacts and the scatterer is localized at its true position after eight iterations. Also, the amplitude of the scatterer is closer to the true one and it is found to be around 0.87 .

\section{HORIZONTAL LAYERS EXAMPLE}

In this example, the grid spacing $\Delta x=\Delta z=7 \mathrm{~m}$ and the time sampling is $2 \mathrm{~ms}$. Also, the velocity model of this example is shown in Figure 2a. The problem is to recover a subsurface earth model that consists of three horizontal interfaces with three different reflectivities $(0.5,0.25$, and 0.1$)$ as shown in Figure $2 \mathrm{~b}$. We generated 75 shots using the Kirchhoff forward-modeling operator along the surface using a Ricker wavelet with a dominant frequency of $60 \mathrm{~Hz}$.

The standard migration imaging resulted in a blurred version of the true subsurface image as shown in Figure 2c. This is caused by smearing the recorded data over the corresponding migration operator in the image domain (i.e., smearing the recorded event over all possible reflection points). Because we only deployed 75 shots and 75 receivers along the surface, this image is heavily smeared and dominated by the migration smiles leading to this fuzzy depiction of the true reflectivity model. Also, the quality of the migrated image deteriorates considerably near the edges of the model due to the limited acquisition coverage; hence, these regions are dominated by migration artifacts.

To alleviate these problems, LSM is applied to migrate the data and the result in Figure 2d shows the mitigation of the migration artifacts and the enhancement of image quality and the resolution of the reflectors after 30 gradient descent iterations. Furthermore, the least-squares solution partially resolves the reflectors close to the edges of the model and partially compensates for the low illumination near these areas. Nevertheless, LSM still provides a smooth solution, whereas the true reflectivity distribution is sparse a)

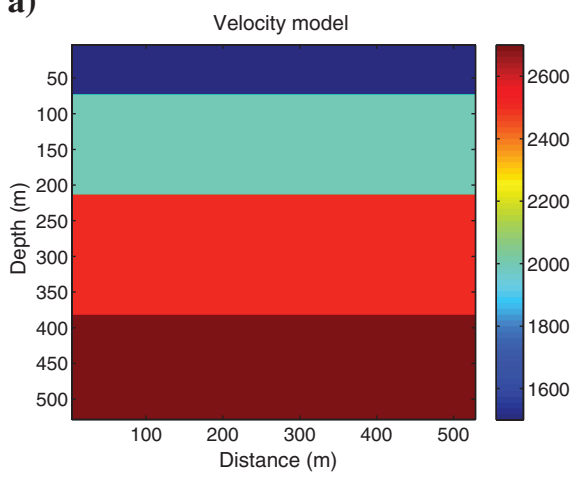

d) b)

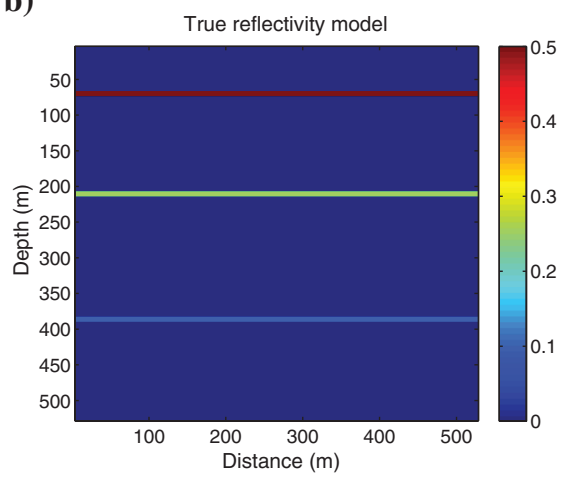

c)

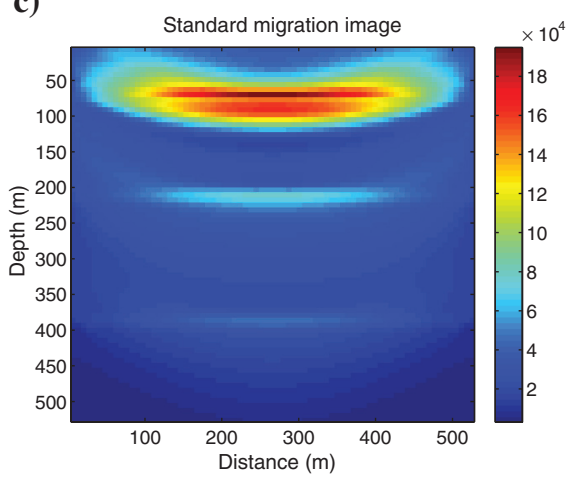

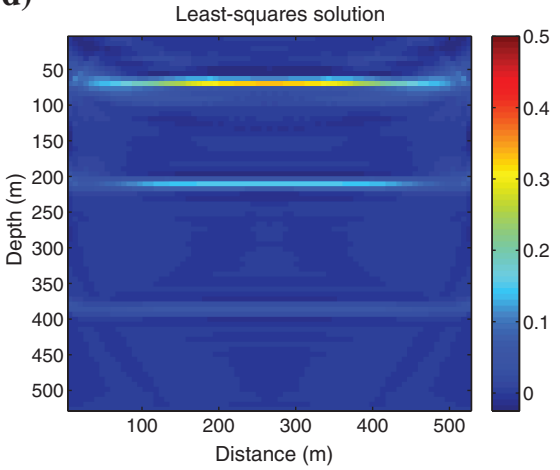

e)

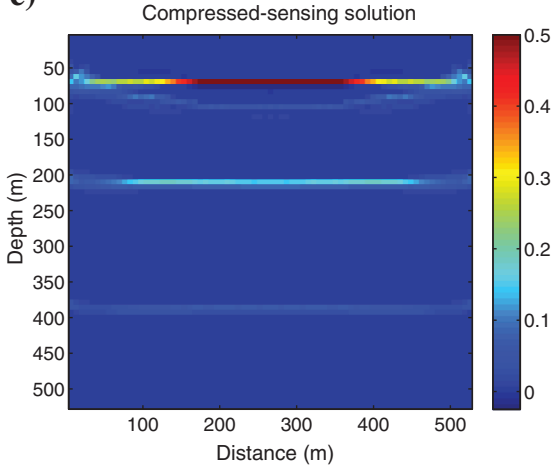

Figure 2. Horizontal layers example: (a) velocity model, (b) true subsurface reflectivity distribution with three reflectors, and (c) blurred standard migrated image. The reflectors are poorly resolved especially near the edges of the model. (d) LSM enhances the spatial resolution and suppresses the smearing artifacts, but the energy of each reflector is smeared over neighboring depth samples. (e) The CS solution focuses the reflectors, suppresses the migration artifacts, and gives accurate amplitude information especially close to the higher coverage area. 
and discontinuous. Note that there exist some spurious artifacts (i.e., events) in the vicinity of each reflector. Also, the amplitude of each reflector is weaker than the true one because the energy is still spread over several depth samples close to the reflector.

The CS solution is obtained by iteratively solving the BPDN problem and is shown in Figure 2e. After 30 iterations and using a value of $\sigma=1 \times 10^{-4}$, this sparse solution shows that the migration artifacts, especially at the edges, are significantly reduced. Also, the amplitude of each reflector is better recovered than the smooth least-squares solution, especially away from the low coverage areas close to the edges of the model.

\section{FAULT MODEL EXAMPLE}

In this example, the grid spacing $\Delta x=\Delta z=10 \mathrm{~m}$, and the time sampling is $2 \mathrm{~ms}$. We computed the standard, unconstrained leastsquares, and CS solutions for a fault model of the earth. The velocity model and the true reflectivity model are shown in Figure $3 \mathrm{a}$ and $3 \mathrm{~b}$, respectively. We generated 75 noise-free shots using the Kirchhoff forward-modeling operator along the surface using a Ricker wavelet with a dominant frequency of $20 \mathrm{~Hz}$.

The standard migration imaging computed a heavily blurred depiction of the true subsurface image as shown in Figure $3 \mathrm{c}$. In fact, it is quite hard to interpret and estimate an accurate position of the fault plane from this image. Moreover, the poor illumination and the limited acquisition aperture worsen the migrated image quality near the edges of the model.

Applying unconstrained LSM to the synthetic data enhances the image quality. One could now delineate the position of the fault plane after 30 gradient descent iterations as clearly seen in Figure 3d. However, we managed to preserve the sparsity nature of the migrated image by solving the BPDN problem and the solution after 30 iterations is shown in Figure $4 \mathrm{a}$. By choosing $\sigma=4 \times 10^{2}$, this sparse solution resolves the events spatially more accurately with more precise amplitude information than the smooth least-squares solution.

An interesting challenge is to find out if one can reconstruct an accurate representation of the subsurface reflectivity model using a fewer number of measurements. For this matter, we significantly reduced the number of sources and receivers by a factor of 5 compared with the previous case (i.e., using only $4 \%$ of the available data). In this case, there are only 15 shots and 15 receivers equally spaced along the surface. The first shot is placed at the top left corner grid point of the model, and the last one is located at the top right corner. The spacing interval between sources and also receivers is now $50 \mathrm{~m}$ instead of $10 \mathrm{~m}$ in the dense geometry.

Although the common-depth-point (CDP) sampling is $5 \mathrm{~m}$ in the dense geometry, it is $25 \mathrm{~m}$ in this sparse acquisition geometry. In the fault model, the velocity ranges from 2000 to $3500 \mathrm{~m} / \mathrm{s}$. With a dominant frequency of $20 \mathrm{~Hz}$, the dominant wavelength ranges from 100 to $175 \mathrm{~m}$. Using the dense geometry, we sampled the image point at 20 to 30 points per wavelength, which is quite dense. On the other hand, with the sparse acquistivity model. ition geometry, the imaging point is sampled at only 4-7 points per wavelength.

The CS solution using the new geometry is shown in Figure 4b, and it is remarkably better than the standard migrated image and the least-squares image using the dense geometry shown in Figure $3 \mathrm{c}$ and $3 \mathrm{~d}$, respectively. The CS solution using the sparse geometry is quite comparable with the CS solution using the dense acquisition geometry shown in Figure 4a even though the image is slightly blurred and the amplitude information of the reflectors becomes less accurate. Hence, with only $4 \%$ of the available data and resources, CS managed to retrieve a highly focused migrated image. The computational time is reduced significantly compared to computing solutions with the dense acquisition geometry.

We also tested CS based-imaging using a more sparse acquisition geometry. In this test, there are only five shots and five receivers equally spaced along the earth surface. Similarly, the first shot is placed at the top left corner grid point of the model and the last one is located at top right corner. Also, the spacing interval between shots and between receivers is now $150 \mathrm{~m}$; hence, the CDP sampling is $75 \mathrm{~m}$. Therefore, the imaging point is sampled only at around one to two points per wavelength. The capability of CS migration deteriorates heavily in this very sparse acquisition geometry as shown in Figure 4c. Thus, the CS imaging requires sufficient CDP sampling of the imaging point to be able to focus the scatterers in their accurate positions.

\section{ROBUSTNESS TO OBSERVATION NOISE}

Another question of interest is to study whether sparse reconstruction is robust in the presence of white Gaussian random a)

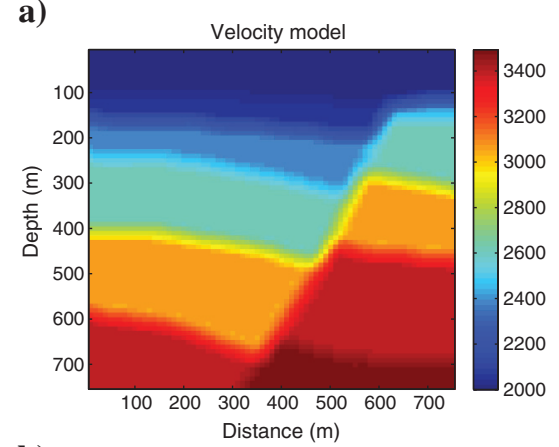

b)

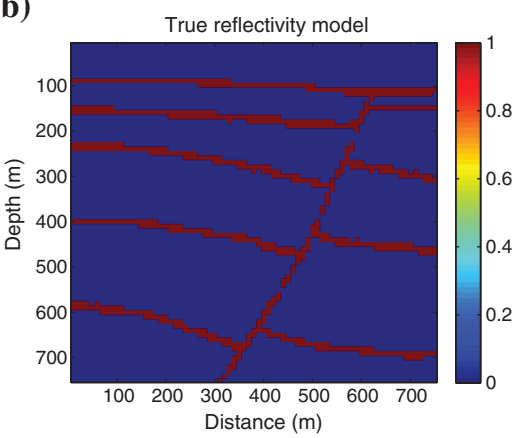

c)

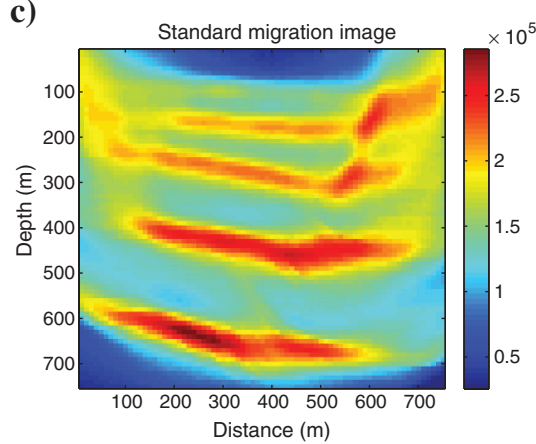

d)

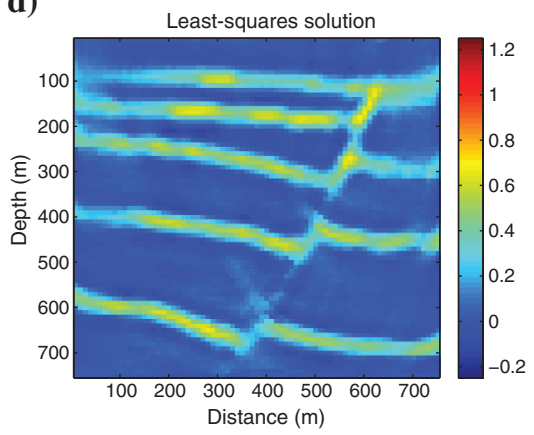

Figure 3. Fault model example: (a) smooth velocity model, (b) true subsurface reflectivity distribution, (c) blurred standard migrated image (it is difficult to delineate the fault plane accurately), and (d) LSM enhances the spatial resolution and suppresses the smearing artifacts but still gives a smooth and dispersed version of the true reflec- 
observation noise. In other words, What would be the effect of observational noise on the image quality?

To answer this question, we added white Gaussian random noise to the synthetic noise-free scattered data used in the fault model example with the dense acquisition geometry. The white Gaussian random noise distribution has a zero mean and a variance of $\gamma^{2}$, and about $68 \%$ of the noise lies within one standard deviation $(\mathrm{STDV}=1 \gamma)$ away from the mean.

We used the CS algorithm to image different data sets with STDVs $\gamma$ equal to 5, 10, and 40. Given that the signal-to-noise ratio $(\mathrm{S} / \mathrm{N})$ is the average power of the signal divided by the average power of the noise, these data sets have S/Ns of 9, 2.27, and 0.142 , respectively. The migrated images for these different variances are shown in Figure 5.

In the case of the smallest STDV, the CS solution in Figure 5a is quite similar to the CS solution in the noise-free case shown in Figure 4a. However, when the STDV is equal to 10, the solution plotted in Figure $5 \mathrm{~b}$ tends to be smoother but it is not as heavily blurred as the noise-free least-squares solution in Figure $3 \mathrm{~d}$ and it has more accurate amplitude information. The robustness of the CS algorithm deteriorates dramatically as shown in Figure $5 \mathrm{c}$ when the average noise power is larger than the average power of the signal $(\mathrm{S} / \mathrm{N} \approx 0.142)$. This represents an extreme case in which the forward-modeling operator fails to explain the considerable amount of noise living in its null space.

\section{ROBUSTNESS TO VELOCITY ERROR}

Building an accurate velocity model and estimating the right velocity for migration are what make the seismic imaging problem quite difficult. For this reason, we need to assess the robustness of the sparsely recovered solution in the presence of velocity error.

Therefore, we applied CS migration to the recorded data using the dense acquisition geometry with velocity models that are $3 \%$ and $5 \%$ faster than the exact velocity model. The results are plotted in Figure $6 \mathrm{a}$ and $6 \mathrm{~b}$, respectively. The reflectors are fairly spatially focused in Figure 6a compared with the defocused events in Figure $6 \mathrm{~b}$. Also, the two figures show that the fault plane is mispositioned and the reflectors, especially the deeper ones, are pushed down.

As expected, the reflectors, especially the deeper ones, are pulled up when using a $3 \%$ and $5 \%$ slower migration velocity to apply the CS migration as shown in Figure 6c and 6d, respectively.

Henceforth, CS-based migration still retrieves fairly sparse migrated images even though the seismic events get defocused and mispositioned when the velocity error increases. Similarly, a)

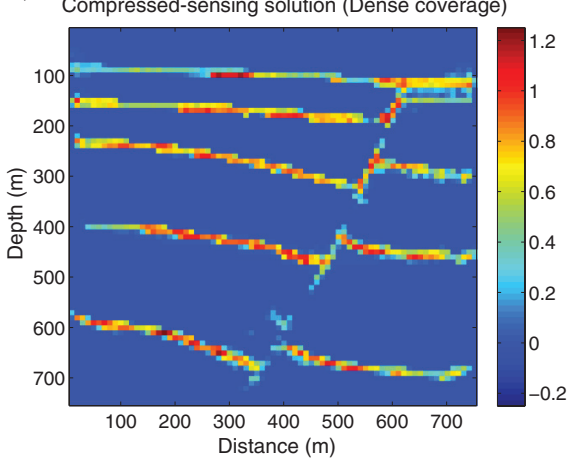

b)

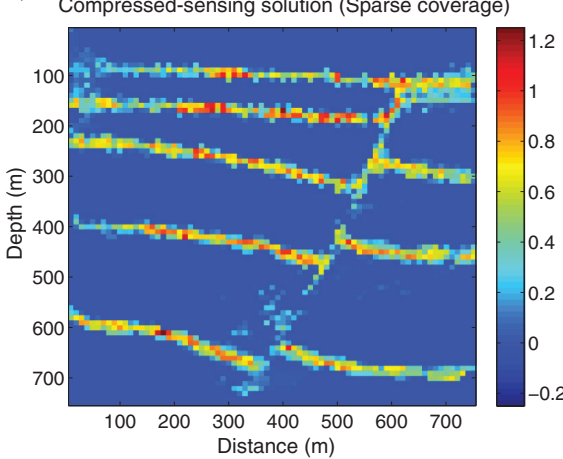

c)

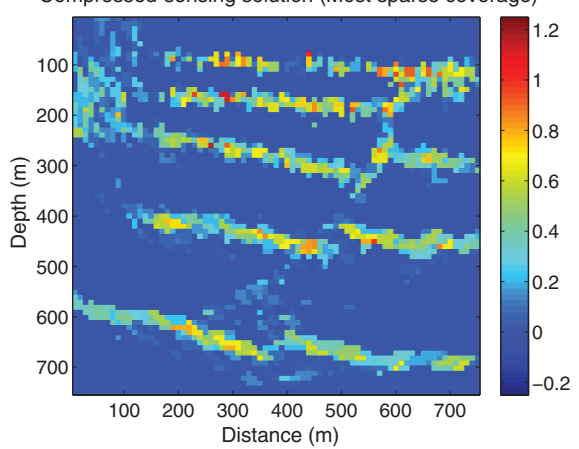

Figure 4. Fault model example: (a) The CS solution using all 75 sources and receivers provides a highly focused migrated image with more accurate amplitude information. (b) The CS solution using only $4 \%$ of the available sources and receivers gives a reasonably compressed and focused migrated image. (c) The CS solution with only five sources and five receivers shows the deterioration of the capability of the CS migration to resolve the image.

a)

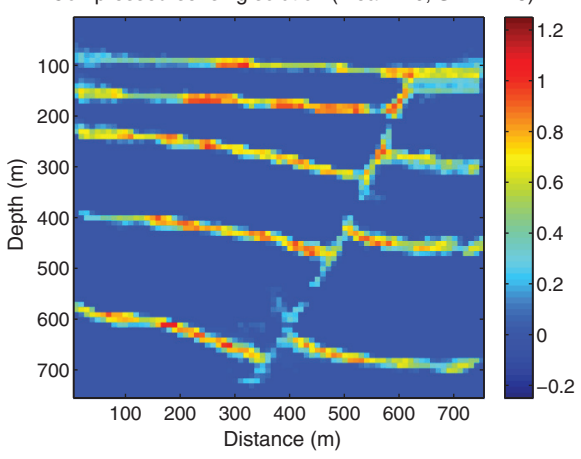

b)

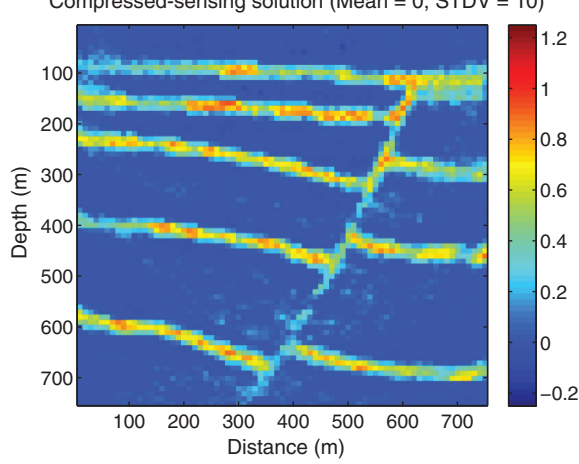

c)

Compressed-sensing solution (Mean $=0, \mathrm{STDV}=40)$

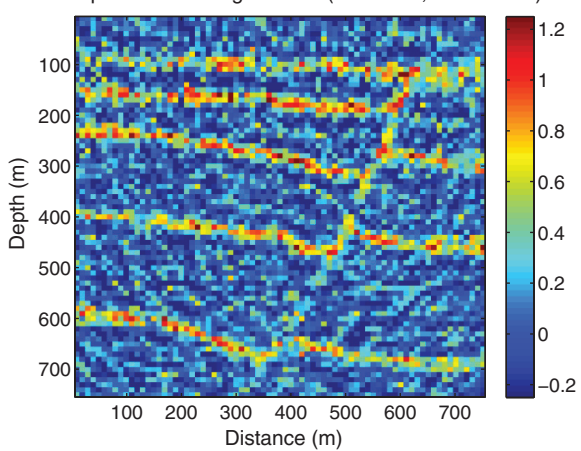

Figure 5. The CS solutions obtained from the noisy data after the addition of white Gaussian random noise with different STDVs: (a) $\gamma=5$, (b) $\gamma=10$, and (c) $\gamma=40$. 
we obtain the least-squares solutions using the erroneous velocities and these are shown in Figure 7 for comparison. Clearly, these solutions are strongly obscured by the migration smiles, and the reflectors are considerably defocused compared with the CS solution.

\section{CONCLUSIONS}

Standard migration suffers from migration artifacts and low spatial resolution due to the limited aperture, coarse source-receiver sampling, band-limitedness nature of the source wavelet, and a)

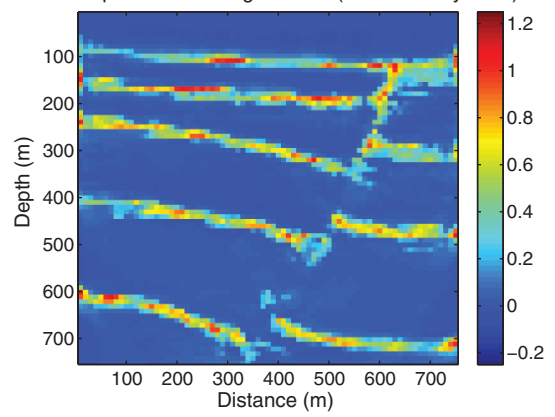

b)

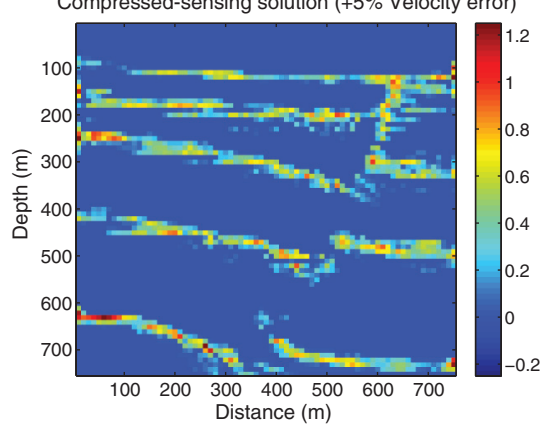

a)

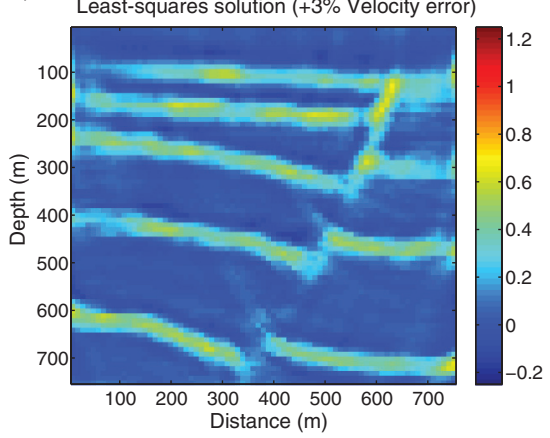

b)

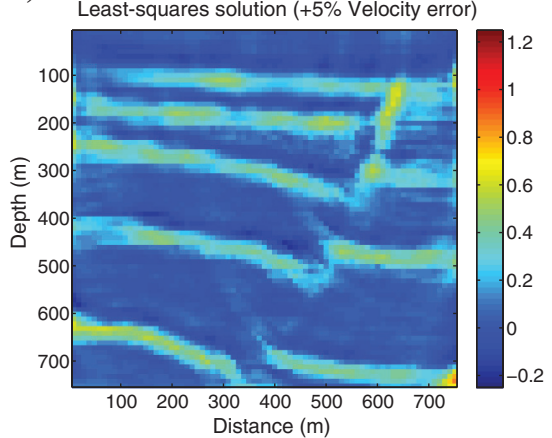

c)

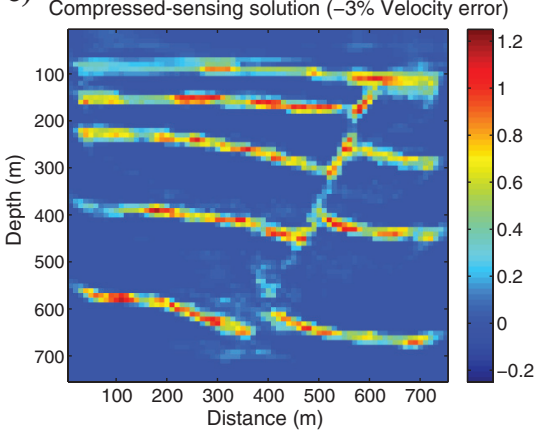

d)

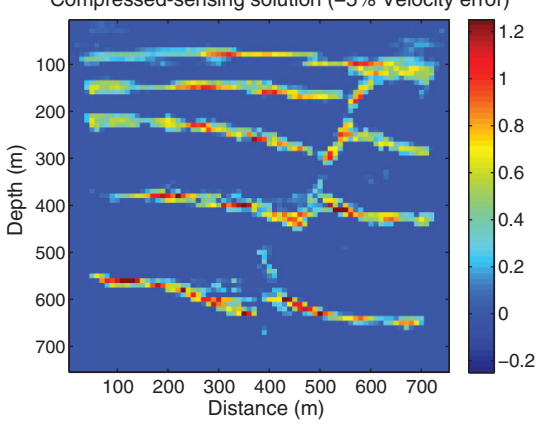

Figure 6. The CS images using inaccurate migration velocities: (a) $3 \%$ faster velocity, (b) $5 \%$ faster velocity, (c) $3 \%$ slower velocity, and (d) $5 \%$ slower velocity.

c)

c) Least-squares solution ( $-3 \%$ Velocity error)

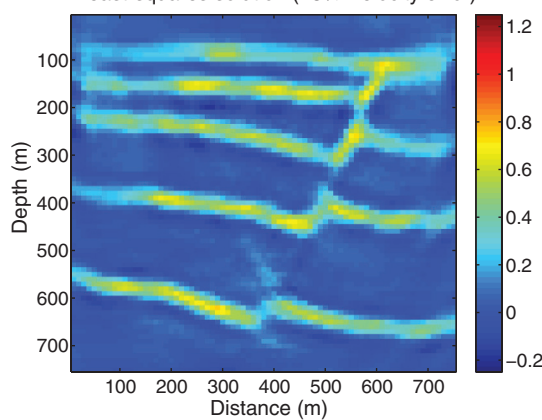

d)

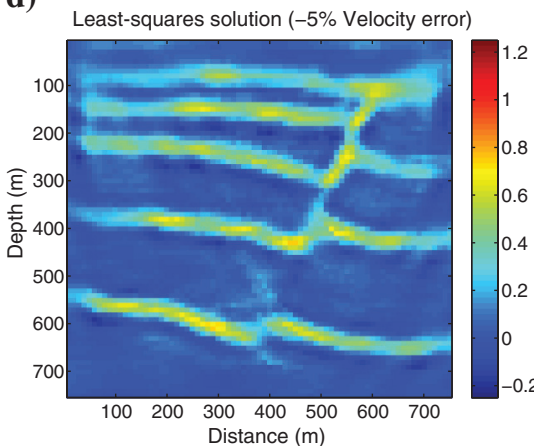

Figure 7. The LSM images using inaccurate migration velocities: (a) 3\% faster velocity, (b) 5\% faster velocity, (c) 3\% slower velocity, and (d) $5 \%$ slower velocity. 
low subsurface illumination. These standard images obtained by applying the adjoint operator managed to retrieve a blurred depiction of the true subsurface reflectivity distribution.

The LSM algorithm helps suppress migration artifacts and enhance the spatial resolution of the migrated section by iteratively finding a subsurface reflectivity model that explains the observed data in a least-squares sense. However, its solution might still be smooth whereas the subsurface reflectivity distribution is inherently sparse.

Formulating the Kirchhoff modeling-migration problem as a BPDN problem has proven to be quite useful. Solving the BPDN problem managed to recover a sparse subsurface reflectivity model that explains the data with more accurate amplitude information.

The CS algorithm was tested on different subsurface models, and it showed that CS solutions significantly reduce the migration artifacts, enhance the spatial resolution, and recover more accurate amplitudes of the reflectors and scatterers than the smooth leastsquares solutions. The CS algorithm dramatically compresses the smearing artifact caused by the adjoint operator and places much of the energy at the corresponding subsurface point scatterer.

Also, by deploying only $20 \%$ of the available sources and $20 \%$ of the available receivers, CS migration retrieves a fairly focused and accurate subsurface reflectivity model using a smaller number of measurements. This could potentially lead to huge savings in resources and computational time. We also demonstrated the robustness of CS in the presence of white Gaussian random noise. Overall, the CS algorithm still retrieves a sparse reflectivity model if the noise variance is small. The quality of the image deteriorates and becomes smoothed and less sparse as we increase the noise variance resulting in migrated images that are comparable to the noise-free least-squares solutions.

We finally demonstrated that the CS migration is quite robust when using inaccurate migration velocities. Using CS migration, we were able to recover fairly sparse migrated images even though the seismic events become more defocused and mispositioned as we used more erroneous migration velocities.

We finally conclude that CS-based migration provides an efficient algorithm to recover sparse representations of subsurface reflectivity distributions. Although one of its major limitations is the sensitivity to errors in migration velocity, it tends to promote sparsity in the solution. So, we would expect the CS solution to be more relevant than standard migration or LSM solutions if the true signal is sparse. In future work, we will consider applying CS-based imaging to a real data example and also to a complex salt synthetic model using a reverse-time migration framework instead of Kirchhoff migration.

\section{ACKNOWLEDGMENTS}

The Spectral Projected Gradient (SPGL1) MATLAB toolbox is a large-scale solver of the BPDN problem, which we used to reconstruct the sparse subsurface reflectivity distributions.

\section{REFERENCES}

Aster, R., B. Borchers, and C. Thurber, 2005, Parameter estimation and inverse problems: Academic Press, International Geophysics.

Avron, H., A. Sharf, C. Greif, and D. Cohen-Or, 2010, $l 1$-sparse reconstruction of sharp point set surfaces: ACM Transactions on Graphics, 29, 135:1-135:12, doi: 10.1145/1857907.1857911.

Chen, S., D. Donoho, and M. Saunders, 2001, Atomic decomposition by basis pursuit: SIAM Review, 43, 129-159, doi: 10.1137/ S003614450037906X.

Claerbout, J. F., 1992, Earth sounding analysis: Processing versus inversion: Blackwell Scientific Publications, Inc.

Claerbout, J. F., and S. Fomel, 2008, Image estimation by example: Geophysical soundings image construction: Multidimensional autoregression: Stanford University.

Crase, E., A. Pica, M. Noble, J. McDonald, and A. Tarantola, 1990, Robust elastic nonlinear waveform inversion: Application to real data: Geophysics, 55, 527-538, doi: 10.1190/1.1442864.

Donoho, D., 2006, Compressed sensing: IEEE Transactions on Information Theory, 52, 1289-1306, doi: 10.1109/TIT.2006.871582.

Eldar, Y., and G. Kutyniok, 2012, Compressed sensing: Theory and applications: Cambridge University Press.

Fomel, S., and A. Guitton, 2006, Regularizing seismic inverse problems by model reparameterization using plane-wave construction: Geophysics, $\mathbf{7 1}$ no. 5, A43-A47, doi: 10.1190/1.2335609.

Landa, E., W. Beydoun, and A. Tarantola, 1989, Reference velocity model estimation from prestack waveforms: Coherency optimization by simulated annealing: Geophysics, 54, 984-990, doi: 10.1190/1.1442741.

LeBras, R., and R. Clayton, 1988, An iterative inversion of backscattered acoustic waves: Geophysics, 53, 501-508, doi: 10.1190/1.1442481.

Lustig, M., D. L. Donoho, and J. M. Pauly, 2006, Rapid MR imaging with "compressed sensing" and randomly under-sampled 3DFT trajectories: Presented at 13th Annual Meeting of the International Society for Magnetic Resonance in Medicine.

Lustig, M., J. M. Santos, J. Hyung Lee, D. L. Donoho, and J. M. Pauly, 2005, Application of compressed sensing for rapid MR imaging: Presented at Signal Processing with Adaptive Sparse Structured Representations Workshop.

Nemeth, T., C. Wu, and G. Schuster, 1999, Least-squares migration of incomplete reflection data: Geophysics, 64, 208-221, doi: 10.1190/1 .1444517.

Pennebaker, W. B., and J. L. Mitchell, 1992, JPEG: Still image data compression standard, 1st ed.: Kluwer Academic Publishers.

Ribodetti, A., S. Operto, W. Agudelo, J. Collot, and J. Virieux, 2011, Joint ray + Born least-squares migration and simulated annealing optimization for target-oriented quantitative seismic imaging: Geophysics, 76, no. 2, R23-R42, doi: 10.1190/1.3554330.

Roth, G., and A. Tarantola, 1992, Inversion of seismic waveforms using neural networks: 62nd Annual International Meeting, SEG, Expanded Abstracts, 788-791.

Snieder, R., M. Y. Xie, A. Tarantola, and A. Pica, 1988, Retrieving both the impedance variations and background velocity in reflection seismics using least-squares waveform inversion: 58th Annual International Meeting, SEG, Expanded Abstracts, 1070-1072.

Tang, Y., 2009, Target-oriented wave-equation least-squares migration/ inversion with phase-encoded Hessian: Geophysics, 74, no. 6, WCA95WCA107, doi: 10.1190/1.3204768.

Taubman, D., and M. Marcellin, 2002, JPEG2000: Image compression fundamentals, standards, and practice: Springer-Verlag GmbH, The Springer International Series in Engineering and Computer Science.

Trzasko, J., and A. Manduca, 2009, Highly undersampled magnetic resonance image reconstruction via homotopic $l_{0}$-minimization: IEEE Transactions on Medical Imaging, 28, 106-121, doi: 10.1109/TMI.2008 .927346 .

van den Berg, E., and M. P. Friedlander, 2007, SPGL1: A solver for largescale sparse reconstruction, http://www.cs.ubc.ca/labs/scl/spgl1, accessed 1 September 2012.

van den Berg, E., and M. P. Friedlander, 2008, Probing the Pareto frontier for basis pursuit solutions: SIAM Journal on Scientific Computing, 31, 890-912, doi: 10.1137/080714488.

Yu, S., L. Tranchevent, and Y. Moreau, 2011, Kernel-based data fusion for machine learning: Methods and applications in bioinformatics and text mining: Springer, Studies in Computational Intelligence. 